\title{
Design of Simulation for Time-Division Multiplexing Digital Optimal Frequency Band Transmission System
}

\author{
Luyong Ren*, Qian Xi \\ College of Computer Science and Technology, Shandong University of Technology, Zibo, China \\ Email: ^renly@sdut.edu.cn
}

How to cite this paper: Ren, L.Y. and Xi, Q. (2021) Design of Simulation for TimeDivision Multiplexing Digital Optimal Frequency Band Transmission System. Open Journal of Applied Sciences, 11, 1083-1091. https://doi.org/10.4236/ojapps.2021.1110080

Received: August 11, 2021

Accepted: October 10, 2021

Published: October 13, 2021

Copyright ( 2021 by author(s) and Scientific Research Publishing Inc. This work is licensed under the Creative Commons Attribution International License (CC BY 4.0).

http://creativecommons.org/licenses/by/4.0/

\section{Open Access}

\begin{abstract}
This paper designs a simulation experiment model of the overall structure of time-division multiplexing digital optimal frequency band transmission system based on MATLAB simulation platform. The parameters of each module in the simulation model are set. The working process and performance of the time-division multiplexing digital optimal band transmission system are simulated. The simulation results show that the digital optimal band transmission system achieves the best transmission receiving conditions and performance, and the designed time-division multiplexing optimal digital band transmission simulation system achieves its functions. The research in this paper will help to improve the level of digital communication technology and to understand the structure of time-division multiplexing digital optimal band transmission system.
\end{abstract}

\section{Keywords}

Time Division Multiplexing, Optimal Reception, Frequency Band Transmission System, Matlab, Simulink Simulation

\section{Introduction}

With the rapid development of Global Science and Technology, the development of digital communication is more and more rapid. Digital communication technology has penetrated into all kinds of communication services. In modern digital communication, the long-distance transmission of digital signal needs to pass through the band Communication Channel, that is to say, the frequency-band transmission of digital signal is more widely used. Therefore, in the theory of communication principle, digital band transmission system 
theory, system structure, performance analysis, system design and other content are one of the important theories of communication principle. However, there are factors such as inter-symbol interference and noise interference in the channel transmission of digital signals, which cause the reliability of the system to decrease. In addition, the actual digital communication system generally uses multiplexing technology to improve the communication system transmission efficiency. Therefore, the actual digital communication system is a time-division multiplexing digital frequency band transmission system with the characteristics of large transmission capacity, eliminating inter-symbol interference and achieving optimal reception (maximum output signal-to-noise ratio). The system, which includes the theory of crosstalk and the theory of optimal receiver, is a practical communication system with complex structure and practical application environment. We use MATLAB [1] [2] to build the simulation system of the time-division multiplexing digital optimal frequency band transmission system, with the help of the simulation design and analysis of the time-division multiplexing digital optimal frequency band transmission system, so that we deeply understand the theoretical knowledge of communication technology, and master the use of development simulation platform. Therefore, the experimental simulation analysis platform for the establishment of a complete structural and comprehensive functions time division multiplexed digital optimal band transmission system is the purpose of this study, and the experimental simulation platform can verify the realistic communication technology. Moreover, the simulation platform can save resources, shorten the time cycle and reduce the development cost in the research and development of real communication system, which will lay a foundation for the future research and development of communication system.

\section{Composition and Principle of Transmission Systems}

\subsection{Constitution of the System}

The structure of the time division multiplexed digital optimal frequency band transmission system is shown in Figure 1. It consists of multiple signal inputs, a time division multiplexer, a transmission pulse shaping filter with the square root characteristic of the raised cosine spectrum, a digital modulator. Besides, it is composed of communication channel, digital demodulator, receiving matched filter with square root characteristics of raised cosine spectrum, sampling

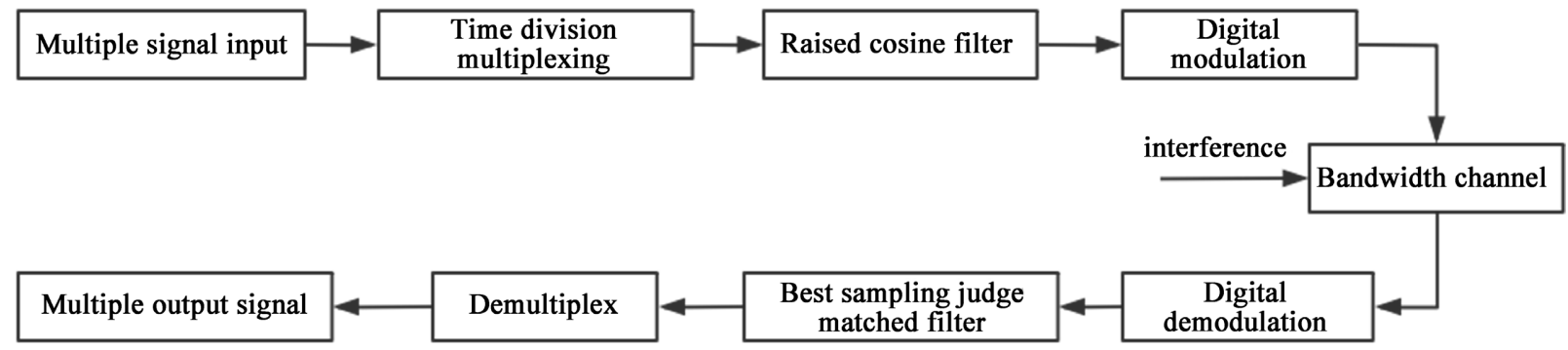

Figure 1. Block diagram of time division multiplexing digital optimal frequency band transmission system. 
decision circuit, time division multiplexer, and multiple signal output.

\subsection{System Implementation Optimal Transmission}

In the circuit structure of the transmission system shown in Figure 1, the band-pass transmission system is composed of a digital modulator, a communication channel, and a digital demodulator. In order to achieve the best reception of the digital frequency band transmission system, the receiving filter should be designed as a matched filter, and the pulse shaping transmission filter before the digital modulator should be designed as a filter with the square root characteristic of the raised cosine spectrum.

Principle analysis: Let $G_{T}(f)$ be the transfer function of the pulse-shaping filter, $G_{R}(f)$ is the transfer function of the receiving filter, $C(f)$ is the transmission characteristic of the digital modulation and demodulation band communication channel, under ideal conditions $C(f)=1$; The total transfer function of the system composed of $G_{T}(f), G_{R}(f)$ and $C(f) H(f)=G_{T}(f) \cdot G_{R}(f) \cdot C(f)$; To eliminate inter-code crosstalk, $H(f)$ must meet Nyquist's first criterion. At the same time, to achieve the best reception, $G_{R}(f)$ must be the complex conjugate relationship of $G_{T}(f)$, that is, $\left|G_{R}(f)\right|=\left|G_{T}(f)^{*}\right|$ So the expressions of $\left|G_{R}(f)\right|, G_{T}(f)$ satisfy formula (1) [3] [4]

$$
|H(f)|=\left|G_{R}(f)\right| \cdot|C(f)| \cdot\left|G_{T}(f)\right|=\left|G_{R}(f)\right|^{2}=\left|G_{T}(f)\right|^{2}
$$

can be written as:

$$
\left|G_{R}(f)\right|=\left|G_{T}(f)\right|=\sqrt{|H(f)|}
$$

Therefore, Formula (2) shows that the transmit and receive filters are designed with the square root characteristics of the raised cosine spectrum to achieve the best reception.

The system composed of pulse shaping transmitting filter, bandpass transmission system, and receiving matched filter can be called equivalent baseband channel transmission system. This equivalent baseband channel transmission system achieves the best reception with the maximum output signal-to-noise ratio at the output of the matched filter, and the total transmission function of its corresponding digital frequency band transmission system conforms to the $\mathrm{Ny}$ quist criterion, which means that Therefore, the crosstalk between the sampled time codes is zero. At the same time, the total transfer function $H(f)$ of the system is designed as a raised cosine roll-off transfer function, which is physically achievable. If the channel is not an ideal channel, the inter-symbol crosstalk of the transmission system is not completely eliminated, and an equalizer can be added to further eliminate the inter-symbol crosstalk. Therefore, the data of multiple signal sources are connected to high-speed data through time division multiplexing, the system, which is then transmitted through the optimal frequency band transmission system, is the Time-division multiplexing digital optimal frequency band transmission system, which eliminates inter symbol interference and achieves optimal reception. 


\section{Simulation Design Based on Matlab-Based Transmission System}

\subsection{Design of Time-Division Multiplexing Module}

In the design, the input signal of the Time-division multiplexing is a four-way signal, which is designed to be a square wave, a sawtooth wave and two sine waves with different amplitudes, and then set their parameters. Using the Subsystem module, the simulation model of the designed Time-division multiplexing module is shown in Figure 2.

This module realizes that the four input signals are extracted and digitized sequentially according to time, and then the signals extracted from different time slots are merged into a multi-channel signal through the merging module. At the receiving end, the multiplexed signal will be separated into the original four signals through the Subsystem 1 module, and the multiplexed and demultiplexed waveforms will be displayed on the oscilloscope.

\subsection{Design of Digital Modulator/Demodulator Module}

The digital modulation/demodulation method is designed as 2PSK, in which the 2PSK signal is first generated by keying method, and then demodulated by coherent demodulation method [5] [6] [7] [8] The modulated signal and the Additive white Gaussian noise signal are then added together to produce a modulated signal with noise, and the input signal is a time division multiplexing signal. The simulation circuit model of the designed digital modulator/demodulator is shown in Figure 3.

Parameter setting:

Carrier signal parameters: Amplitude 3, initial phase "0" and "180"; Sampling time $0.001 \mathrm{~s}$.

\subsection{Design of Transmit Filter, Matched Filter, Channel and Sampling Decider Module}

1) Transmission filter and matched filter adopt Discrete Filter module filter

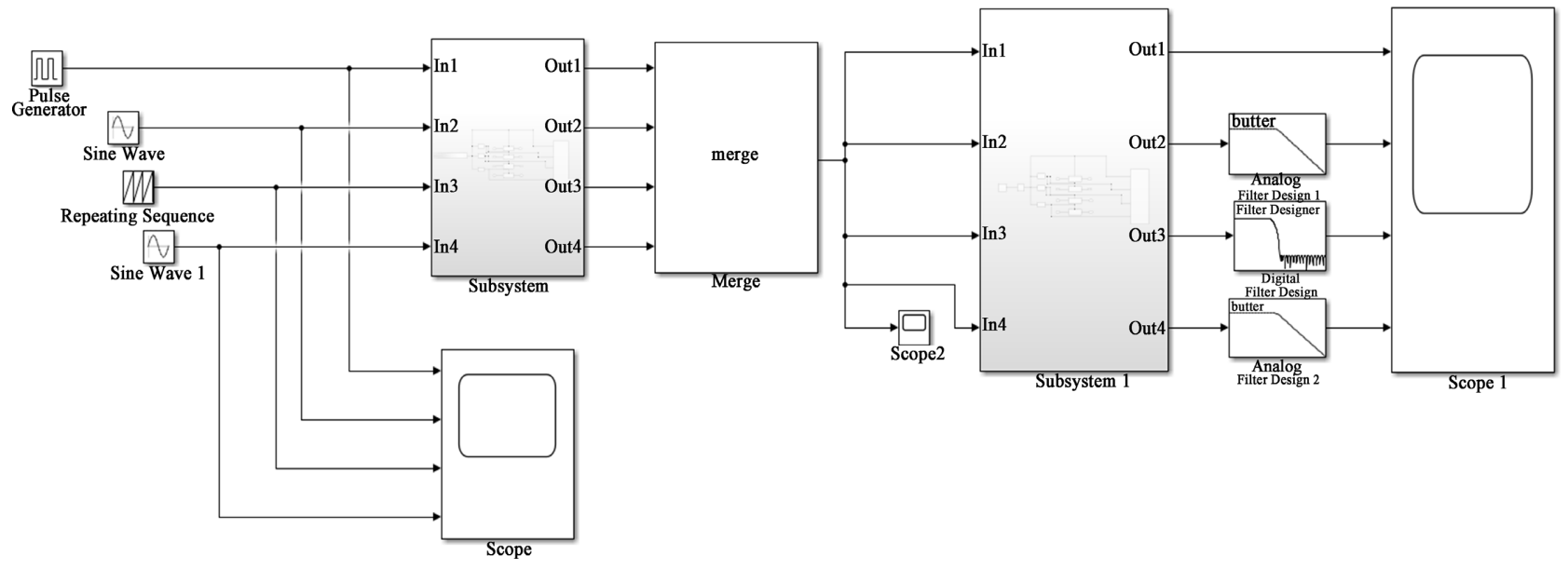

Figure 2. Time division four-channel multiplexing/demultiplexing simulation module. 


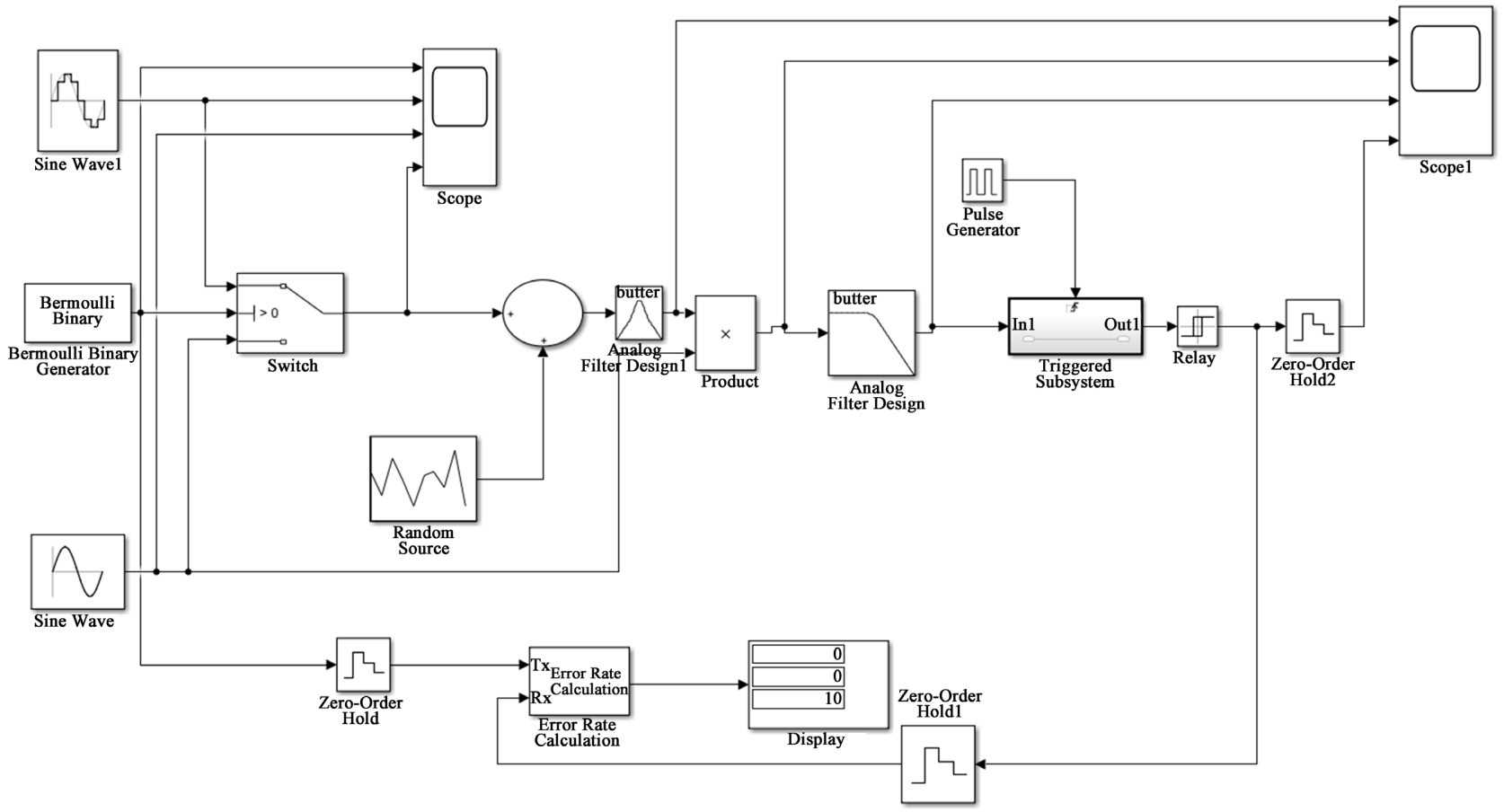

Figure 3. Simulation circuit model of 2PSK digital modulator/demodulator.

with square root raised cosine characteristics, its parameters are set to "Numerator" to "rcosine $(1,10$, 'fir/sqrt', $0.5,10)$ ", where 0.5 is the roll-off factor, "Denominator" is "1", "Sample time" is " 0.001 ".

2) The Channel "AWGN Channel" module uses an additive white Gaussian noise channel with parameters "EB/No $(\mathrm{dB})$ " " 15 " and "Symbol period" is “0.001".

3) The sampling decision is made up of "Product", "Relay", "Enabled and Triggered Subsystem", in which "Relay" parameter "Output w' hen on" is " 3 ", "Output when off" is " -3 ", sampling time is the frequency of multiplex signal sequence.

\subsection{System Simulation Model Implementation}

The simulation circuit model of Time Division multiplex digital optimal band Transmission System is composed of Time-division multiplexing module, transmit filter module, 2PSK modulation module, channel, 2PSK demodulation module, matched receiving filter module, time division multiplexing module, etc., the model block diagram is shown in Figure 4.

\section{Simulation and Analysis of Transmission Systems}

\subsection{Transmission System Simulation}

Four-way source input signal parameter setting: First, amplitude 3, period 2, no delay square wave; second, amplitude 3, period pi sine wave; third, Amplitude 2, period 2 sawtooth wave; fourth, amplitude 2, period 2 * pi sine wave.

The time domain waveform is shown in Figures 5(a)-(d). The other main 
modules according to the unit module design parameters, and then adjust the debugging simulation system. Adjustment complete, running simulation results.

\subsection{Analysis of Simulation Results of Transmission System}

System simulation in the corresponding oscilloscope display results, oscilloscope display results: The input time-domain waveform of the signal is shown in Figures 5(a)-(d). The output time-domain waveform after TDM is shown in Figure 6. The time-domain waveform of the modulated signal is shown in Figure 7. The optimal sampling time of the matched filter is shown in Figure 8, the

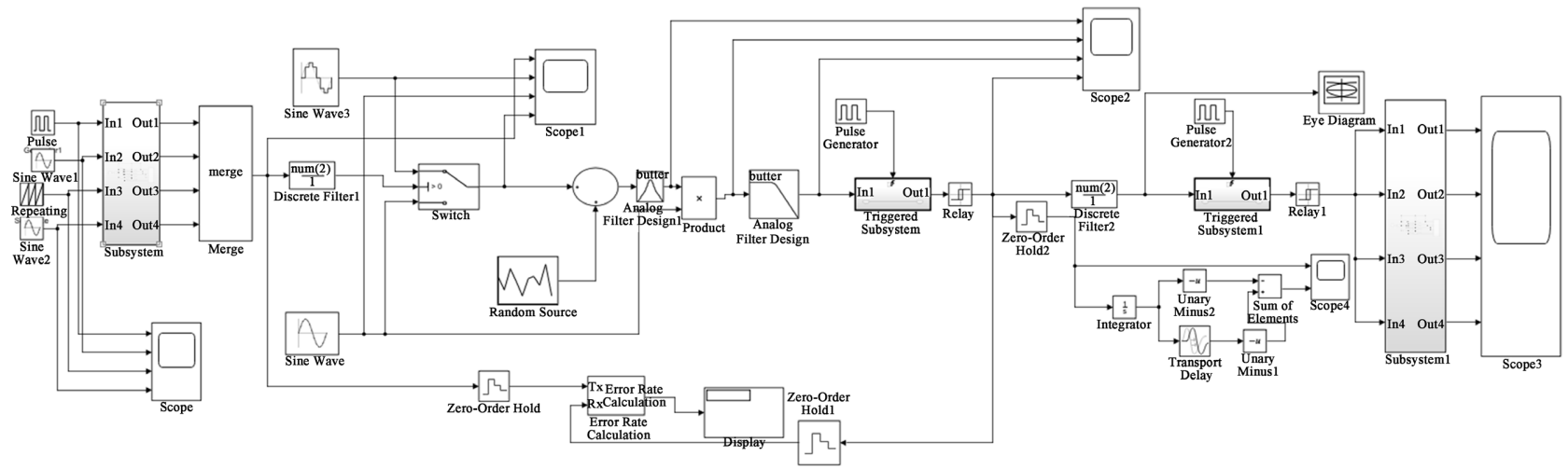

Figure 4. Simulation model of time-division multiplexing digital best band transmission system.

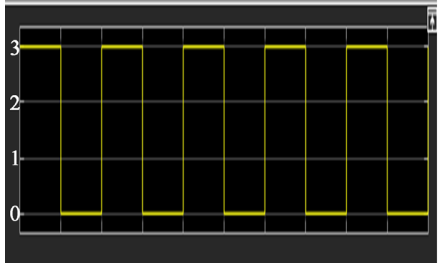

(a)

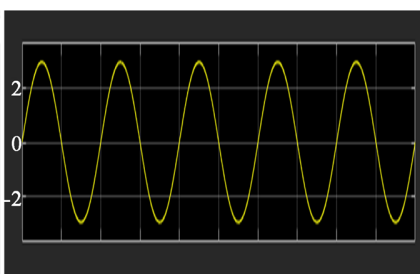

(b)

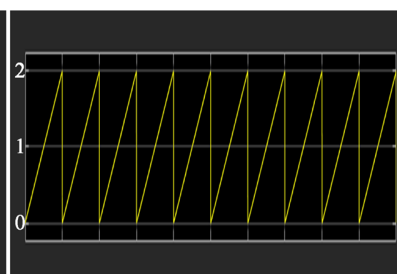

(c)

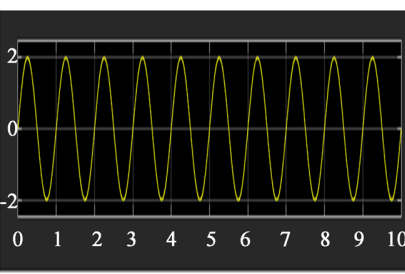

(d)

Figure 5. Four-way input.

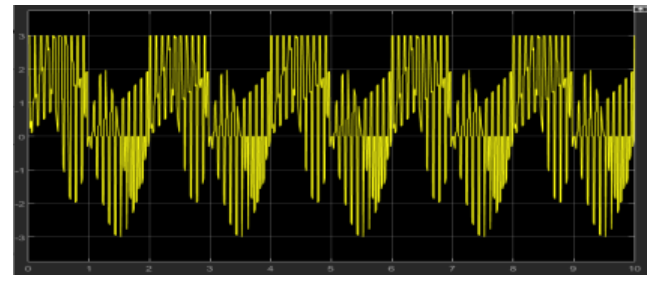

Figure 6. Signal waveform behind the time-division multiplexing.

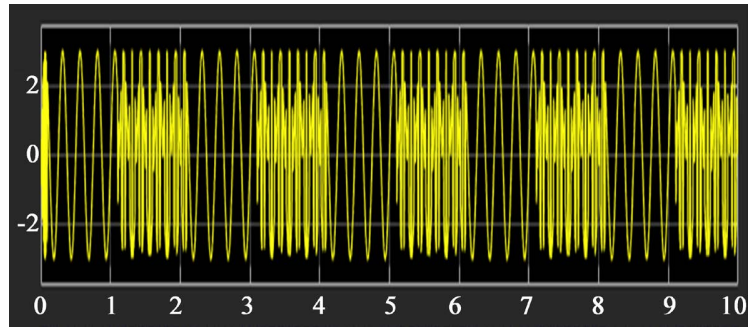

Figure 7. 2PSK modulated back waveform. 


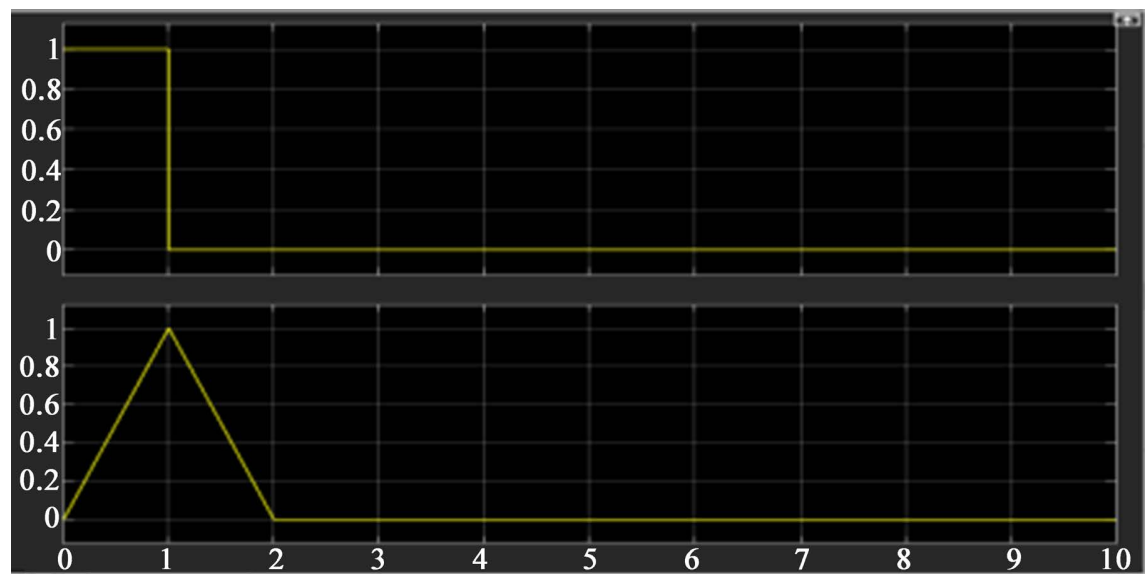

Figure 8. Matched filter sampling time.

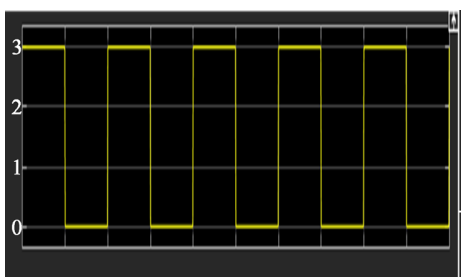

(a)

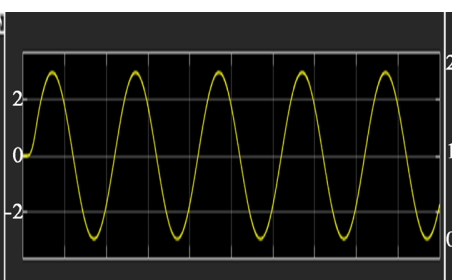

(b)

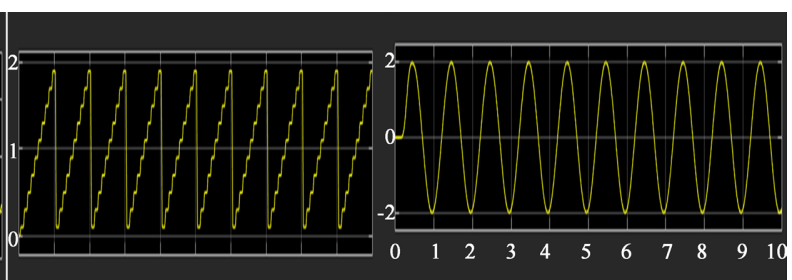

(c) (d)

Figure 9. Four-channel output waveform after demultiplexing.

demultiplexing output time domain waveform of the system is shown in Figures 9(a)-(d).

The simulation results show that the waveform of the original signal is the same as that of the input signal. The bit error rate (Ber) of the simulation system is 0 , and the eye diagram of the simulation system is clear, which shows that the matched filter can restrain the noise and ISI to some extent. So we can see that the design of the whole system is reasonable and the digital frequency band transmission system achieves the best receiving state. The simulation results verify that the designed time-division multiplexing has completed its functions and met its design requirements. The simulation research results of this article will help us understand how to optimize and design the time division multiplexed digital optimal frequency band transmission system in practical applications, it has important application value.

\section{Conclusion}

The simulation verifies the correctness of the structure and complete functions of the designed time-division multiplex digital optimal frequency band transmission simulation experiment system. In the theoretical knowledge of communication principles, the content of each link of the time division multiplex digital optimal frequency band transmission system basically contains all the main content of the communication principle theory, understands and masters the theoretical content and working process of each link of the time division mul- 
tiple digital optimal frequency band transmission system. Performance parameters, and design process also have a more comprehensive grasp of communication principles and technology. Therefore, the purpose of designing the simulation experiment system of time division multiplexing digital optimal frequency band transmission system is to allow students and enthusiasts studying communication technology to use our existing laboratory conditions and the designed simulation system to do it themselves. Practice designing this complex communication system yourself. In the design process, the process from theory to practice, from simple to complex to innovation is realized. In the actual design of students, some communication technologies used in actual communication: such as analog signal digitization technology, time division multiplexing, band-pass transmission, optimal reception, synchronization technology and other technologies can be used for deep theoretical understanding and simulation design realization research. In short, through this simulation design experimental system applied to experimental teaching, people can deeply understand the structure, performance and principle of time division multiplexing digital best frequency band transmission system. The theory is very helpful and improves the effect.

\section{Conflicts of Interest}

The authors declare no conflicts of interest regarding the publication of this paper.

\section{References}

[1] Al-Rawi, M. (2019) Data-Aided Carrier Recovery with QPSK Modulation. Scientific Bulletin, 24, 14-22. https://doi.org/10.2478/bsaft-2019-0002

[2] He, F. (2019) Simulation and Analysis of MSK System based on SystemView. International Journal of Intelligent Information and Management Science, 8, 62-66.

[3] Chai, J. and Nie, Y. (2017) Research and Analysis of Digital Baseband Transmission System Based on MATLAB. Journal of Secure Communication and System, 1, Article ID: 516 . https://doi.org/10.18063/jscs.v1i1.516

[4] Omijeh, B.O. and Eyo, I. (2016) Comparative Study of Bit Error Rate of Different M-ary Modulation Techniques in AWGN Channel. American Journal of Networks and Communications, 5, 82-90. https://doi.org/10.11648/j.ajnc.20160505.11

[5] Rajesh, V. and Abdul Rajak, A.R. (2020) Channel Estimation for Image Restoration Using OFDM with Various Digital Modulation Schemes. Journal of Physics: Conference Series, 1706, Article ID: 012076. https://doi.org/10.1088/1742-6596/1706/1/012076

[6] Ahmed, Md.F., Ahmed, Md.F. and Md. Touhidul Islam, A.Z.. (2021) Comparison of Bit Error Rate Performance of Various Digital Modulation Schemes over AWGN and Rayleigh Fading Channels Using Simulink. The International Journal of Ambient Systems and Applications, 9, 7-16. https://doi.org/10.5121/ijasa.2021.9202

[7] Wang, G. and Cheng, W. (2017) Analysis and Simulation of Digital Baseband Transmission System Basing on SIMULINK. Proceedings of the 2016 2nd International Conference on Materials Engineering and Information Technology Applications (MEITA 2016), Atlantis Press, 370-374. 
https://doi.org/10.2991/meita-16.2017.77

[8] Balarabe, A.T., et al. (2021) A Comprehensive Analysis of M-ary PSK and M-ary QAM Schemes. Asian Journal of Research in Computer Science, 7, 63-71. https://doi.org/10.9734/ajrcos/2021/v7i330183 